\title{
Hausdorff and packing measure for thick solenoids
}

\author{
by \\ Michą Rams (Warszawa and Dijon)
}

\begin{abstract}
For a linear solenoid with two different contraction coefficients and box dimension greater than 2, we give precise formulas for the Hausdorff and packing dimensions. We prove that the packing measure is infinite and give a condition necessary and sufficient for the Hausdorff measure to be positive, finite and equivalent to the SBR measure. We also give analogous results, generalizing $[\mathrm{P}]$, for affine IFS in $\mathbb{R}^{2}$.
\end{abstract}

1. Introduction. We continue our research $([\mathrm{RS}])$ of relations between the SBR measure and the geometric (Hausdorff and packing) measures for hyperbolic diffeomorphisms with two different negative Lyapunov exponents. Our method is similar to one used in $[\mathrm{P}]$. In $[\mathrm{RS}]$ we dealed with linear Smale-Williams solenoids with box dimension smaller than 2. Here we will consider the case when the box dimension is greater than 2 , i.e. the map is

$$
\phi:(t, x, z) \mapsto\left(k t \bmod 1, \lambda_{1} x+f(t), \lambda_{2} z+g(t)\right),
$$

where $\lambda_{2}<\lambda_{1}<1 / 2, k \lambda_{1}>1$ and $k \lambda_{1} \lambda_{2}<1$. The map is defined on the torus $T=S^{1} \times D, D$ being the unit disk. The functions $f, g$ are smooth $\left(C^{2}\right)$ and chosen in such a way that $\phi$ is an injection.

Let $\Lambda$ be the attractor of $\phi$. Define

$$
s=2+\frac{\ln k+\ln \lambda_{1}}{-\ln \lambda_{2}} .
$$

The SBR measure of $\phi$ will be denoted by $\mu$. The projection of $\mu$ onto the $(t, x)$ plane will be denoted by $\nu$. As proved lately by Tsuji in [T], for generic choice of $f$ and $g, \nu$ is absolutely continuous with respect to Lebesgue measure and its density is in $L^{2}$. It is an open problem whether the density of $\nu$ is bounded.

The main results of this paper are as follows:

Theorem 1.1. The packing dimension of $\Lambda$ is s. For generic $f, g$ the s-dimensional packing measure of $\Lambda$ is infinite.

2000 Mathematics Subject Classification: Primary 28A78.

Supported by Foundation for Polish Science. 
Theorem 1.2. For generic $f, g$ the Hausdorff dimension of $\Lambda$ is s. For generic $f, g$ if the density of $\nu$ is unbounded then the s-dimensional Hausdorff measure of $\Lambda$ is zero, otherwise it is positive, finite and equivalent to $\mu$.

For the definitions and properties of the Hausdorff and packing dimensions and measures we refer the reader to $[\mathrm{M}]$.

The paper is divided as follows. In the second section we explain the notation and give the basic properties of the solenoid map we are going to use. The third section contains the proofs of Theorems 1.1 and 1.2. In the last section we apply the same method to investigate affine IFS in $\mathbb{R}^{2}$.

A word about notation: the symbol $c$ stands for any constant and need not denote the same constant in another formula.

The author wishes to thank Maciej Wojtkowski for his help with language problems.

2. Solenoid map. The image of the torus $T$ under $\phi$ is topologically a torus again. The plane $\{t=0\}$ cuts $\phi(T)$ into $k$ sets that will be called first level cylinders. One of them is the image of all points in $T$ with $t$ coordinate between 0 and $1 / k$, another one is the image of points with $t$ coordinate between $1 / k$ and $2 / k$ and so on. Similarly, this plane cuts $\phi^{n}(T)$ into $k^{n}$ $n$th level cylinders. Note that the image of an $n$th level cylinder under $\phi$ is the union of $k(n+1)$ st level cylinders, lying one in each of the first level cylinders. The $n$th level cylinders are geometrically (roughly) elliptic cylinders of height 1 and diameters $\lambda_{1}^{n}$ and $\lambda_{2}^{n}$.

As $\phi(T) \subset T$, the attractor $\Lambda$ is contained in $\phi^{n}(T)$ for all $n$. It is locally a Cantor bouquet of (disjoint) curves. Each of those curves is the infinite intersection of a decreasing family of cylinders of increasing levels. The curves are graphs of smooth functions $(x(t), z(t))$ so the angle between them and the planes $\{t=$ const $\}$ is bounded away from zero. The set $\pi(\Lambda)$ is a Cantor bouquet of (intersecting) curves as well. For a generic solenoid map all these intersections are transversal ([B], $[\mathrm{S}])$ so if the intersecting curves come from different $n$th level cylinders, the angle between them at the intersection point is bounded away from 0 by a constant depending only on $n$.

As the projection $\pi$ and the solenoid map $\phi$ commute, one can define $\widehat{\phi}=\pi \circ \phi \circ \pi^{-1}$. Locally this mapping is contracting $\lambda_{1}$ times in the $x$ direction and expanding $k$ times in the $t$ direction. The set $\pi(\Lambda)$ is invariant under $\widehat{\phi}$.

We may write the measure $\mu$ as

$$
\mu=\int \mu_{t} d t
$$

where $\mu_{t}$ is the $(1 / k, \ldots, 1 / k)$-Bernoulli measure on the Cantor set in the 
stable section of the solenoid. Similarly,

$$
\nu=\int \nu_{t} d t
$$

The measures $\mu_{t}, \nu_{t}$ count basically how many curves intersect a given set. As the curves are graphs, we may write

$$
\nu_{t \pm \varepsilon}([a+K \varepsilon, b-K \varepsilon]) \leq \nu_{t}([a, b]) \leq \nu_{t \pm \varepsilon}([a-K \varepsilon, b+K \varepsilon]),
$$

where $K$ is a constant not depending on $\varepsilon$.

Two easy geometric lemmas follow immediately:

LEMma 2.1. Let $R$ be a rectangle

$$
R=\left\{(t, x) ; t_{0}-b<t<t_{0}+b, x_{0}-a<x<x_{0}+a\right\}
$$

and let $2 b K<a$. Choose $d_{1}, d_{2}<a / K-b$ and let $A, B$ be two hexagons given by

$$
\begin{array}{r}
A=\left\{(t, x) ; t_{0}-d_{1}<t<t_{0}+d_{2},\right. \\
\left.x_{0}-a+K\left(\left|t-t_{0}\right|+b\right)<x<x_{0}+a-K\left(\left|t-t_{0}\right|+b\right)\right\}, \\
B=\left\{(t, x) ; t_{0}-d_{1}<t<t_{0}+d_{2},\right. \\
\left.x_{0}-a-K\left(\left|t-t_{0}\right|+b\right)<x<x_{0}+a+K\left(\left|t-t_{0}\right|+b\right)\right\} .
\end{array}
$$

Then

$$
\frac{2 b}{d_{1}+d_{2}} \nu(A) \leq \nu(R) \leq \frac{2 b}{d_{1}+d_{2}} \nu(B) .
$$

LeMma 2.2. At almost all points $(t, x)$,

$$
\frac{d \nu}{d \mathrm{Leb}^{2}}(t, x)=\frac{d \nu_{t}}{d \mathrm{Leb}^{1}}(x),
$$

where Leb ${ }^{2}$, Leb ${ }^{1}$ stand for the Lebesgue measures in $\mathbb{R}^{2}$ and $\mathbb{R}^{1}$, respectively.

3. Proofs of Theorems $\mathbf{1 . 1}$ and 1.2. Let us start from an easy proposition. Note that it is true for every, and not almost every, solenoid map.

Proposition 3.1. The box dimension of $\Lambda$ equals s. The s-dimensional Hausdorff measure of $\Lambda$ is finite.

Proof. We use the ellipsoid cutting partition, that is, we divide every $n$th level cylinder into $\lambda_{1}^{n} \lambda_{2}^{-2 n}$ approximate balls of size $\lambda_{2}^{n}$.

We proceed with the proofs of Theorems 1.1 and 1.2. We are working with a generic solenoid map in the rest of this section. We may assume that $\Lambda$ is strictly contained in $T$ - this is a generic property.

We are going to use the Frostman Lemma. We will compute the upper and lower $s$-density of the measure $\mu$ at almost every point of $\Lambda$. We are not interested in the precise value, we only care whether the density is positive 
and whether it is finite. Hence we may freely replace in the definition of $s$ density the ball $B_{r}(x)$ by an arbitrary set containing $B_{c r}(x)$ and contained in $B_{r / c}(x)$. We may choose the same set for a different point $y$ lying at a distance not greater than $\mathrm{cr} / 2$ from $x$ - so such two points will be equivalent for us while computing the $r$-approximation of the $s$-density. We will call these the freedom of choice (FOC) properties.

Let $(t, x, z) \in \Lambda$ and denote by $C(t, x, z ; n)$ the $n$th level cylinder containing $(t, x, z)$. Let $K_{r}(t, x, z)$ be a cube of side $r$ centered at $(t, x, z)$. As $\Lambda$ is at some distance $d$ from $\partial T$, it is at distance at least $\lambda_{2}^{n} d$ from $\partial\left(\phi^{n}(T)\right)$. Hence

$$
\begin{aligned}
K_{c \lambda_{2}^{n}}(t, x, z) \cap \Lambda & \subset K_{\lambda_{2}^{n}}(t, x, z) \cap C(t, x, z ; n) \cap \Lambda \\
& \subset K_{\lambda_{2}^{n}}(t, x, z) \cap \Lambda
\end{aligned}
$$

for some $c$ not depending on $(t, x, z)$ nor on $n$.

We set $Z_{n}(t, x, z)=K_{\lambda_{2}^{n}}(t, x, z) \cap C(t, x, z ; n) \cap \Lambda$. The SRB measure is preserved under $\phi$, so the measure of $Z_{n}(t, x, z)$ is equal to the measure of its $n$th preimage. The set $\phi^{-n}\left(Z_{n}(t, x, z)\right)$ is the intersection of $\Lambda$ and an approximate orthogonal parallelepiped of sides $\lambda_{2}^{n} k^{-n} \times \lambda_{1}^{-n} \lambda_{2}^{n} \times 1$, centered at $\phi^{-n}(t, x, z)$. The measure $\mu$ of this set is thus equal to the measure $\nu$ of its projection onto the $(t, x)$ plane, i.e. $\pi(\Lambda)$ intersected with the rectangle $R_{n}(t, x, z)$ of sides $\lambda_{2}^{n} k^{-n} \times \lambda_{1}^{-n} \lambda_{2}^{n}$, centered at $\pi\left(\phi^{-n}(t, x, z)\right)$.

Note that the area of $R_{n}(t, x, z)$ is equal to $\lambda_{2}^{n s}$. By FOC we may write

$$
\begin{aligned}
& \bar{D}_{s}(\mu)(t, x, z) \approx \limsup _{n \rightarrow \infty} \frac{1}{\lambda_{2}^{n s}} \mu\left(Z_{n}(t, x, z)\right)=\limsup _{n \rightarrow \infty} \frac{\nu\left(R_{n}(t, x, z)\right)}{\operatorname{Leb}\left(R_{n}(t, x, z)\right)}, \\
& \underline{D}_{s}(\mu)(t, x, z) \approx \liminf _{n \rightarrow \infty} \frac{1}{\lambda_{2}^{n s}} \mu\left(Z_{n}(t, x, z)\right)=\liminf _{n \rightarrow \infty} \frac{\nu\left(R_{n}(t, x, z)\right)}{\operatorname{Leb}\left(R_{n}(t, x, z)\right)} .
\end{aligned}
$$

By Lemma 2.1,

$$
\begin{aligned}
\lambda_{1}^{n} k^{-n} \nu\left(B_{c_{1} \lambda_{1}^{-n} \lambda_{2}^{n}}\right. & \left.\left(\pi \circ \phi^{-n}(t, x, z)\right)\right) \\
\leq & \nu\left(R_{n}(t, x, z)\right) \leq \lambda_{1}^{n} k^{-n} \nu\left(B_{c_{2} \lambda_{1}^{-n} \lambda_{2}^{n}}\left(\pi \circ \phi^{-n}(t, x, z)\right)\right),
\end{aligned}
$$

where $c_{1}$ and $c_{2}$ depend only on $K$ and do not depend on $t, x, z, n$.

Hence (by FOC) we may replace (3.2) and (3.3) by

$$
\begin{aligned}
& \bar{D}_{s}(\mu)(t, x, z) \approx \limsup _{n \rightarrow \infty} \frac{\nu\left(S_{n}(t, x, z)\right)}{\operatorname{Leb}\left(S_{n}(t, x, z)\right)}, \\
& \underline{D}_{s}(\mu)(t, x, z) \approx \liminf _{n \rightarrow \infty} \frac{\nu\left(S_{n}(t, x, z)\right)}{\operatorname{Leb}\left(S_{n}(t, x, z)\right)},
\end{aligned}
$$

where $S_{n}(t, x, z)=B_{\lambda_{1}^{-n} \lambda_{2}^{n}}\left(\pi \circ \phi^{-n}(t, x, z)\right)$.

The following two observations are crucial to proving Theorems 1.1 and 1.2. First, the function $\nu\left(B_{r}(t, x, z)\right) / \operatorname{Leb}\left(B_{r}(t, x, z)\right)$ for small enough $r$ will 
approximate the density $d \nu / d \operatorname{Leb}(t, x, z)$ for $\nu$-a.e. $(t, x, z)$. Second, the inverse trajectory of $\mu$-almost every point in $\Lambda$ is dense in $\Lambda$.

If the density of $\nu$ is unbounded, one can find a positive $\nu$ measure set $X \subset \pi(\Lambda)$ on which the density is greater than some constant $M$. By the Egorov Theorem one can find a constant $r_{0}$ and a positive $\nu$ measure subset $Y \subset X$ such that if $(t, x) \in Y$ and $r<r_{0}$ then $\nu\left(B_{r}(t, x)\right) \geq$ $M / 2 \operatorname{Leb}\left(B_{r}(t, x)\right)$. The inverse trajectory of $\mu$-almost every point $(t, x, z)$ will meet $\pi^{-1}(Y)$ infinitely many times and for almost all of them $\lambda_{1}^{-n} \lambda_{2}^{n}$ $<r_{0}$. So, by (3.4) the upper $s$-density of $\mu$ is infinite almost everywhere, hence the $s$-dimensional Hausdorff measure is zero.

If the density of $\nu$ is bounded, then so is $\nu\left(B_{r}(t, x, z)\right) / \operatorname{Leb}\left(B_{r}(t, x, z)\right)$, which implies the boundedness of the upper $s$-density of $\mu$ at every point in $\Lambda$. This together with Proposition 3.1 implies that the $s$-dimensional Hausdorff measure of $\Lambda$ is positive and finite.

The following standard argument (see $[\mathrm{P}]$ ) then shows that the Hausdorff measure must be equivalent to the SRB measure. Every $n$th level cylinder is bi-Lipschitz equivalent to any other $n$th level cylinder, and the Lipschitz constant is uniformly bounded, so any two cylinders must have comparable Hausdorff measure. The same holds for any section of an $n$th level cylinder (cut off by planes $\left\{t=t_{0}\right\}$ and $\left\{t=t_{1}\right\}$ ). So, the $s$-dimensional Hausdorff measure of such a section must be approximately equal to $\left(t_{1}-t_{0}\right) k^{-n} H^{s}(\Lambda)$ while its $\mu$ measure equals $\left(t_{1}-t_{0}\right) k^{-n}$.

Note the role of the linearity assumptions in this argument. Both the Hausdorff and SBR measures are equivalent to the maximal entropy measure (one giving equal values to all the cylinders) only because the contraction (for the Hausdorff measure) and expansion (for the SBR measure) coefficients are constant all over the solenoid. For the general solenoid one does not even have an invariant measure of dimension equal to the Hausdorff dimension of the attractor.

LEMMA 3.2. For a generic solenoid map for every $\varepsilon>0$ the upper $(s-\varepsilon)$ density of $\mu$ is zero $\mu$-almost everywhere.

Proof. Let $X$ be a subset of $\Lambda$ such that if $(t, x, z) \in X$ then

$$
\bar{D}_{s-\varepsilon}(\mu)(t, x, z)>M>0 \text {. }
$$

Similarly to (3.4) we have

$$
\bar{D}_{s-\varepsilon}(\mu)(t, x, z) \approx \limsup _{n \rightarrow \infty} \lambda_{2}^{n \varepsilon} \frac{\nu\left(S_{n}(t, x, z)\right)}{\operatorname{Leb}\left(S_{n}(t, x, z)\right)} .
$$

Let $X_{n}$ be the set of points $(t, x, z) \in \Lambda$ for which

$$
\frac{\nu\left(S_{n}(t, x, z)\right)}{\operatorname{Leb}\left(S_{n}(t, x, z)\right)} \geq \lambda_{2}^{-n \varepsilon / 2},
$$

and define $\mu\left(X_{n}\right)=d_{n}$. It is easy to see that $X \subset \lim \sup X_{n}$. 
Divide $\pi(T)=[0,1] \times S^{1}$ into squares $E_{i}$ of side $\lambda_{1}^{-n} \lambda_{2}^{n}$ each. Whenever a point from $\pi \circ \phi^{-n}\left(X_{n}\right)$ belongs to some $E_{i}$, the measure of the square with the same center as $E_{i}$ but three times its size (which we will denote by $F_{i}$ ) is not smaller than $\lambda_{1}^{-2 n} \lambda_{2}^{n(2-\varepsilon / 2)}$, and the union of all $F_{i}$ (which we will denote by $Y_{n}$ ) has $\nu$ measure at least $d_{n}$.

By the Schwarz Lemma,

$$
\int_{F_{i}}\left(\frac{d \nu}{d \operatorname{Leb}}(t, x)\right)^{2} d t d x \geq \frac{\left(\nu\left(F_{i}\right)\right)^{2}}{\operatorname{Leb}\left(F_{i}\right)} .
$$

As any point can belong to at most $9 F_{i}$ 's, we get

$$
\int_{Y_{n}}\left(\frac{d \nu}{d \operatorname{Leb}}(t, x)\right)^{2} d t d x \geq \frac{1}{9} \sum_{i} \int_{F_{i}}\left(\frac{d \nu}{d \operatorname{Leb}}(t, x)\right)^{2} d t d x \geq \frac{1}{81} d_{n} \lambda_{2}^{-n \varepsilon / 2},
$$

where the last inequality becomes an equality if all the $F_{i}$ 's have minimal possible measure, equally distributed on them (by the Schwarz Lemma again).

Hence,

$$
d_{n} \leq 81 N \lambda_{2}^{n \varepsilon / 2}
$$

where

$$
N=\int_{\pi(T)}\left(\frac{d \nu}{d \operatorname{Leb}}(t, x)\right)^{2} d t d x
$$

(it is finite for a generic solenoid map by $[\mathrm{T}]$ ). So, the sequence $d_{n}$ is summable and by the Borel-Cantelli Lemma the set $\lim \sup X_{n}($ and $X$ ) must have $\mu$-measure 0 .

From this lemma we see that the Hausdorff dimension of $\Lambda$ is $s$, which ends the proof of Theorem 1.2.

To prove the packing measure part (Theorem 1.1), note that the lower $s$-density of $\mu$ must be finite $\mu$-almost everywhere. Hence, the $s$-dimensional packing measure of $\Lambda$ is always positive. By Proposition 3.1 the packing dimension of $\Lambda$ cannot be greater than $s$, so it must be $s$.

To complete the proof of Theorem 1.1 we need the following lemma:

Lemma 3.3. For a generic solenoid map, for every $\varepsilon$ there exists a subset $X_{\varepsilon} \subset \pi(\Lambda)$ of positive $\nu$ measure such that the density of $\nu$ is smaller than $\varepsilon$ on $X_{\varepsilon}$.

Proof. Suppose the oposite, i.e. that the density of $\nu$ with respect to Lebesgue measure is either 0 or greater than some $\varepsilon$. The points of positive density of $\nu$ form a positive measure invariant subset of $\pi(\Lambda)$, hence are of full measure and dense in $\pi(\Lambda)$. 
For every $t$ let $a(t)=\max \{x ;(t, x) \in \pi(\Lambda)\}$. Let $Q=\{(t, a(t))\}$; one can easily check that this curve is contained in its image under $\widehat{\phi}$. Let $b(t)=$ $\max \{x ;(t, x) \in \pi(\Lambda),(t, x)$ and $(t, a(t))$ are projections of points from $\Lambda$, belonging to different first level cylinders $\}$.

Let $S=\{(t, x) \in \pi(\Lambda) ; x>b(t)\}$. By transversality, there are only finitely many values (say, $N$ ) of $t$ for which $a(t)=b(t)$. Moreover, again by transversality, if $t_{0}$ is one of these values, then

$$
a(t)-b(t) \geq a\left|t-t_{0}\right|
$$

for some $a$ and for $t$ close to $t_{0}$.

Every point in $S$ has only one preimage under $\widehat{\phi}$. The standard Jacobi formula gives

$$
\frac{d \nu}{d \operatorname{Leb}}(t, x)=\sum_{\left(t^{\prime}, x^{\prime}\right) \in \widehat{\phi}^{-1}(t, x)}\left(k \lambda_{1}\right)^{-1} \frac{d \nu}{d \operatorname{Leb}}\left(t^{\prime}, x^{\prime}\right) .
$$

Hence, if $(t, x) \in S$, then the density of $\nu$ at $(t, x)$ is smaller than at its only preimage.

We will now construct a set of points of Lebesgue measure bounded from below and of arbitrarily high density of $\nu$ measure, which will give a contradiction. Choose $t_{0}$ such that $\operatorname{Leb}^{1}\left(\left\{(t, x) \in \pi(\Lambda) ; t=t_{0}, x \geq a(t)-d\right\}\right)$ $\geq d l$ for some $l$ and all sufficiently small $d$ (almost any $t_{0}$ will do the job). Let $I=\left\{(t, x) \in \pi(\Lambda) ; t=t_{0}, x \geq a(t)-d\right\}$ for some small $d$. This is the intersection of $\pi(\Lambda)$ and an interval of length $d$ with right endpoint in $Q$. At $\nu$-almost all points of $I$ (and at Lebesgue almost all points as well) the density of $\nu$ is greater than $\varepsilon$.

If $I \subset S$, the preimage $\widehat{\phi}^{-1}(I)$ is the intersection of $\pi(\Lambda)$ and an interval of length $\lambda_{1}^{-1} d$ with right endpoint in $Q$. The density of $\nu$ at almost all points of this set is (by (3.7)) greater than $k \lambda_{1} \varepsilon$. In particular, the same is true if we shorten this interval to have length $d$, keeping its right endpoint in $Q$. We repeat this procedure, taking the preimage of the new (shortened) set. We will get a family of sets of the same size and with greater and greater density of $\nu$.

From time to time we will encounter problems, namely when the $t$ coordinate of our set gets too close to one of the $N$ points in $Q \backslash S$. In this case part of our interval may lie outside $S$; we will then take the preimage only of the part of our interval contained in $S$. The length of this part is not shorter than $a \operatorname{dist}(t, Q \backslash S)$ (see (3.6)). As our interval will get shortened in this way, we will not shorten it for some iterations until it grows to the size of $d$. Note that if $d$ was chosen sufficiently small, by (3.6) such close approaches will happen very rarely. Hence we will always return with our interval to the initial size if we iterate long enough, for almost all choices 
of the initial $t_{0}$. We also assume $t_{0}$ was chosen in such a way that we will never hit $Q \backslash S$ directly.

After sufficiently many steps, the set $I^{\prime}=\left\{(t, x) \in \pi(\Lambda) ; t=t_{0}, x \geq\right.$ $a(t)-d\}$ has (one-dimensional) Lebesgue measure not less than $c d$ and the $\nu$ density greater than some $M$. By Lemma 2.2 the $\nu_{t}$ density and $\nu$ density are the same. Hence,

$$
\nu_{t}(a(t)-d, a(t)) \geq d l M
$$

and (by Lemma 2.1)

$$
\nu\left(B_{c d}(t, a(t))\right) \geq d^{2} l M .
$$

For $M$ large enough, $d^{2} l M>1$ and hence the measure of this ball would be greater than the measure of all $\pi(\Lambda)$, which is impossible.

We note again that the projection of the inverse trajectory of almost every point in $\Lambda$ must visit the set of points where the density of $\nu$ is smaller than some $\varepsilon$ infinitely often. Hence, the lower $s$-density of $\mu$ is smaller than $\varepsilon$ almost everywhere and, as $\varepsilon$ was arbitrary, it must be zero. The $s$ dimensional packing measure of $\Lambda$ is thus infinite. This completes the proof of Theorem 1.1.

4. Iterated function systems. In this section we will be working with different systems. Let $f_{1}, \ldots, f_{k}$ be affine contractions in $\mathbb{R}^{2}$ given by

$$
f_{i}(x, y)=\left(\lambda_{1} x+x_{i}, \lambda_{2} y+y_{i}\right)
$$

where $\lambda_{2}<\lambda_{1}<1, k \lambda_{1}>1$ and $k \lambda_{1} \lambda_{2}<1$. We assume that $f_{i}((0,1) \times$ $(0,1)) \cap f_{j}((0,1) \times(0,1))=\emptyset$ for $i \neq j$. These sets will be called first level cylinders, their images under different $f_{i}$ 's are second level cylinders and so on. We denote by $\Lambda$ the unique non-empty compact set satisfying

$$
\Lambda=\bigcup_{i} f_{i}(\Lambda)
$$

Denote $f_{i}^{-1}(x, y)$ by $f(x, y)$. As $f_{i}(\Lambda)$ and $f_{j}(\Lambda)$ are disjoint for $i \neq j, f$ is a well defined map from $\Lambda$ into $\Lambda$.

Let

$$
s=\frac{\log \lambda_{2}-\log \lambda_{1}-\log k}{\log \lambda_{2}} .
$$

Let $\Sigma=\{1, \ldots, k\}^{\mathbb{N}}$. We define a projection $\widehat{\pi}$ from $\Sigma$ onto $\Lambda$ by

$$
\widehat{\pi}(\omega)=\lim _{n \rightarrow \infty} f_{\omega_{n}} \circ \cdots \circ f_{\omega^{1}}(0,0),
$$

where $\omega=\left(\omega_{1} \omega_{2} \cdots\right)$. Let $\widehat{\mu}$ be the $(1 / k, \ldots, 1 / k)$-Bernoulli measure on $\Sigma$. The natural measure of the iterated function system (IFS) is defined as

$$
\mu=\pi_{*}(\widehat{\mu}) .
$$


The projection of $\mu$ in the $y$ direction will be denoted by $\nu$; for generic IFS this measure is absolutely continuous with respect to Lebesgue measure and has $L^{2}$ density (see for example $[\mathrm{R}]$ ). Denote this projection by $\pi$. Let

$$
\widehat{f}_{i}(x)=\pi \circ f_{i} \circ \pi^{-1}(x)=\lambda_{1}(x)+x_{i} .
$$

Our results are as follows:

THEOREM 4.1. For generic IFS satisfying the assumptions above, the box, Hausdorff and packing dimensions of $\Lambda$ are all equal to s. Again for generic IFS, the packing measure of $\Lambda$ is infinite while the Hausdorff measure is positive, finite and equivalent to $\mu$ if and only if the density of $\nu$ with respect to Lebesgue measure is bounded; otherwise the Hausdorff measure of $\Lambda$ is 0 .

Proof. The steps of the proof are very similar to the proofs of Theorems 1.1 and 1.2 (and simpler). Hence we will only sketch it.

Note first that $\Lambda$ may be covered with $k^{n}$ rectangles ( $n$th level cylinders) of sides $\lambda_{1}^{n}$ and $\lambda_{2}^{n}$. As any such rectangle may be covered with $\left(\lambda_{1} / \lambda_{2}\right)^{n}$ balls of radius $\lambda_{2}^{n}$, we immediately prove (similarly to Proposition 3.1 ) that the box dimension of $\Lambda$ equals $s$ and that the $s$-dimensional Hausdorff measure of $\Lambda$ is finite. This was the part of the result that is always true; in what follows we are working with a generic IFS (under the changes of $x_{i}$ 's and $y_{i}$ 's).

We proceed by using the Frostman Lemma. By FOC, as in the previous section, we can use as a neighborhood of $(x, y) \in \Lambda$ a square of side $\lambda_{2}^{n}$, contained in some $n$th level cylinder. The $n$th image under $f$ of such a square is a rectangle of sides $\left(\lambda_{2} / \lambda_{1}\right)^{n}$ and 1 . The $\mu$ measure of this rectangle (equal to $k^{n}$ times the $\mu$ measure of the original square) is thus equal to the $\nu$ measure of its projection in the $y$ direction (being an interval of length $\left.\left(\lambda_{2} / \lambda_{1}\right)^{n}\right)$. Hence,

$$
\underline{D}_{s}(\nu)(x, y) \approx \liminf _{n \rightarrow \infty} \frac{\nu\left(B_{\lambda_{2}^{n} \lambda_{1}^{-n} / 2}\left(\pi \circ f^{n}(x, y)\right)\right)}{\operatorname{Leb}\left(B_{\lambda_{2}^{n} \lambda_{1}^{-n} / 2}\left(\pi \circ f^{n}(x, y)\right)\right)},
$$

and a similar expression holds for $\bar{D}_{s}$.

Similarly to the solenoid case, we thus have to check the properties of the density of $\nu$ with respect to Lebesgue measure. The generic property is that this density exists. The Hausdorff measure part is essentially the same as the proof of Theorem 1.2. The only thing left is the following lemma (analogous to Lemma 3.3).

Lemma 4.2. For every $\varepsilon$ there exists a subset $X_{\varepsilon} \subset \pi(\Lambda)$ of positive $\nu$ measure such that the density of $\nu$ is smaller than $\varepsilon$ on $X_{\varepsilon}$.

Proof. As $x_{i}$ are generically chosen, one may assume that $x_{1}>x_{i}$ for all $i$. Hence there is a set $[a, b] \cap \pi(\Lambda)$ that intersects the projection of only 
one first level cylinder. We may assume that

$$
b=\max \{x ; x \in \pi(\Lambda)\}=\widehat{f}_{1}(x) .
$$

Similarly to (3.7), we have

$$
\frac{d \nu}{d \operatorname{Leb}}(x)=\sum_{i}\left(k \lambda_{1}\right)^{-1} \frac{d \nu}{d \operatorname{Leb}}\left(\widehat{f}_{i}^{-1}(x)\right)
$$

If $x \in[a, b]$, then $\widehat{f}_{i}^{-1}(x) \notin \pi(\Lambda)$ for $i \neq 1$. Hence, for such $x$ the only preimage that can lead to positive density is the one under $\widehat{f}_{1}^{-1}$. If the assertion of the lemma is false, the density of $\nu$ is greater than $\varepsilon$ almost everywhere in $[a, b] \cap \pi(\Lambda)$. Hence, it is greater than $k \lambda_{1} \varepsilon$ almost everywhere in $\widehat{f}_{1}^{-1}([a, b] \cap \pi(\Lambda)) \supset[a, b] \cap \pi(\Lambda)$. This implies that it is greater than $\left(k \lambda_{1}\right)^{2} \varepsilon$ and so on. We conclude that the density of $\nu$ is infinite almost everywhere in $[a, b] \cap \pi(\Lambda)$, which is not possible since this set has positive Lebesgue measure. This contradiction ends the proof.

We finish the proof of Theorem 4.1 like the proof of Theorem 1.1 in the previous section.

\section{References}

[B] H. G. Bothe, The Hausdorff dimension of certain solenoids, Ergodic Theory Dynam. Systems 15 (1995), 449-474.

[M] P. Mattila, Geometry of Sets and Measures in Euclidean Spaces, Cambridge Univ. Press, Cambridge, 1995.

[P] Y. Peres, The packing measure of self-affine carpets, Math. Proc. Cambridge Philos. Soc. 115 (1994), 437-450.

[R] M. Rams, Generic behavior of iterated function systems, Pacific J. Math., to appear.

[RS] M. Rams and K. Simon, Hausdorff and packing measure for solenoids, Ergodic Theory Dynam. Systems 23 (2003), 273-292.

[S] K. Simon, The Hausdorff dimension of the Smale-Williams solenoid with different contraction coefficients, Proc. Amer. Math. Soc. 125 (1997), 1221-1228.

[T] M. Tsuji, Fat solenoidal attractors, Nonlinearity 14 (2001), 1011-1027.

Institute of Mathematics

Polish Academy of Sciences

P.O. Box 21

00-956 Warszawa, Poland

E-mail: rams@impan.gov.pl
Université de Bourgogne Dijon, France 